\title{
Measuring Interphase Stiffening Effects in Styrene-based Polymeric Thin Films
}

Charles D. Wood ${ }^{1}$, Lawrence Chen $^{2}$, Craig Burkhart ${ }^{3}$, Karl Putz ${ }^{1}$, John Torkelson $^{2,4}$, L. Catherine Brinson ${ }^{1,4}$

1 Department of Mechanical Engineering, Northwestern University, 2145 Sheridan Rd, Evanston, IL 60201

${ }^{2}$ Department of Chemical and Biological Engineering, Northwestern University, 2145 Sheridan Rd, Evanston, IL 60201

${ }^{3}$ Goodyear Tire and Rubber Company, Akron, OH 44305

${ }^{4}$ Department of Materials Science and Engineering, Northwestern University, 2220 Campus Drive, Evanston, IL 60201

\section{Abstract}

Incorporating fairly low volume fractions of nanomaterials into polymers creates composites with a vast amount of interfacial area for potential matrix-filler interactions. This surface area provides interfaces between the polymer matrix and the filler at which polymer chains are physically and, potentially, chemically constrained, affecting the local mobility of the polymer chains. In this study, we investigate the properties of this interphase region in several styrene-based polymers and carbon surfaces via model thin film systems. We employ dynamic nanoindentation to probe viscoelastic interphase enhancements in supported thin films for four different polymers with a wide range of glass transition temperatures. The results demonstrate that interphase enhancements depend upon the nature of the substrate/polymer interaction and the average mobility of the polymer, glassy or rubbery. We hypothesize that strongly positive interactions between styrene and graphitic surfaces through pi-pi secondary interactions at the interface create the interphase enhancements we measure and quantify.

\section{Introduction}


Polymers filled with nanomaterials, commonly called nanocomposites, have promised novel materials with enhanced properties such as stiffness, conductivity, toughness, diffusion resistance, and strength[1, 2]. Previous studies on polymer nanocomposites have shown substantial enhancements in macroscale properties with only small volumes of nanomaterial fillers[3-6]. These types of improvements have been attributed to regions of altered mobility within the polymeric matrix phase. These regions of polymer adjacent to the nanofillers are called the 'interphase', which exhibits properties that differ from those of the bulk polymer. At the filler-polymer interface, the polymer potentially interacts with the filler, e.g. van der Waals forces and/or hydrogen bonding, and at the same time is physically confined. This cumulative confinement effect results in property enhancements that can percolate and influence macroscale properties[7]. Including the altered properties of these regions is often necessary in computational models in order to match experimental results.

In order to study the properties of the interphase, one-dimensional model nanocomposites can be used to create thin polymer films on supporting substrates that act as particle mimics, as seen in Figure 1. Previous work with thin films has demonstrated glass transition temperature changes in thin polymeric films $(<300 \mathrm{~nm})$, as well as strong free-surface effects[8-12]. When the probed polymer region is devoid of free-surface effects and fully confined, interphase gradients are shown as strongly positive (increasing the glass transition temperature)[9]. Contact-based methods, such as nanoindentation or atomic force microscopy, are good methods to characterize the mechanical 
properties of the interphase due to their load/displacement sensitivity and spatial resolution. These experiments, through a variety of testing schematics, have provided evidence of modulus/stiffness enhancements in the interphase regime[13, 14]. One approach is to probe the top free-surface of the polymer in proximity to fillers or interfaces in-situ using small radius tips[15-17]. In this case with thin film experiments, the interphase is fully confined and not exposed to the free surface, but results are complicated by the presence of the supporting substrate, as shown in Figure 1. This complex substrate effect can be modeled computationally and then removed from the data through a variety of methods[18-20]. In all of these cases, interphase mechanical properties have demonstrated a power law or exponential decay behavior over a 30-150 nm region, which depends upon the nature of the polymer and substrate interaction[12].
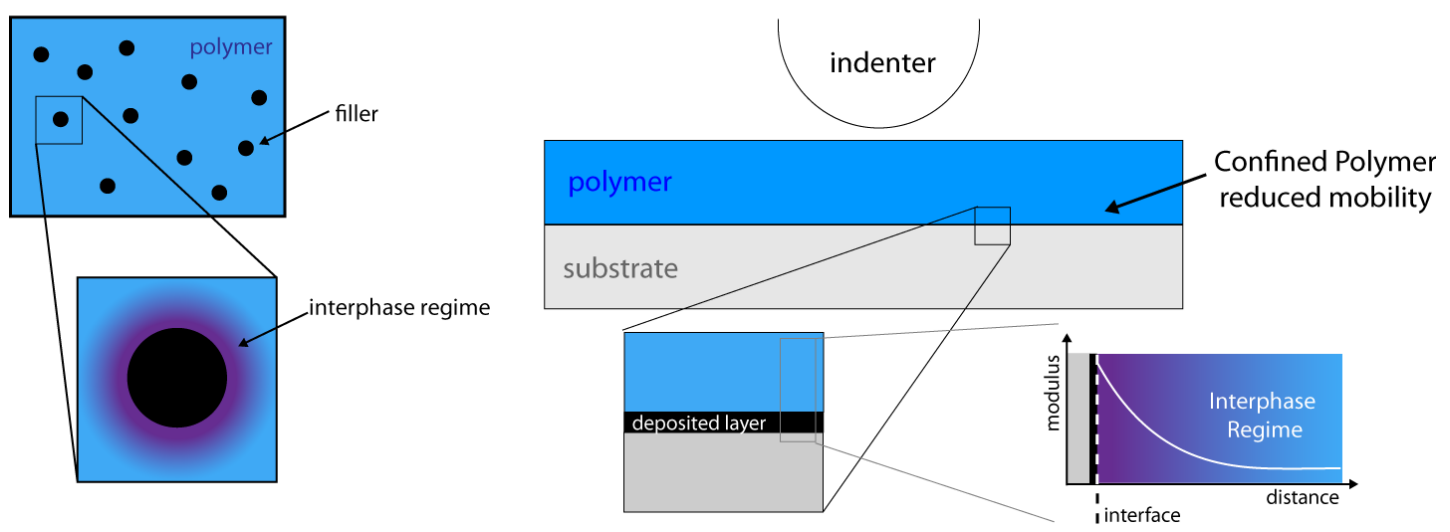

Figure 1. Interphase gradients surrounding particles can be modeled by creating a thin deposited nanoparticle or nanoparticle-mimic layer on a clean substrate. This layer transforms radial interphase gradients into linear gradients across the thickness of the film. This simplification allows the user to probe the top surface and sense the interphase below through indentation experiments. 
Some of the most common nanomaterials used for fillers are carbon based such as carbon nanotubes, graphene, and carbon black. The form of carbon typically defines important factors such as electrical and thermal conductivities, and price. The functional differences between the various forms are attributable to the type of carbon-carbon bonds, particle morphology and chemical purity. Graphitic-like surfaces possess $\mathrm{sp}^{2}$ hybridization of carbon-carbon single bonds; graphene, for instance, has a large number of $\mathrm{sp}^{2}$ bonds, whereas carbon black, an impure but commonly used form, can have varying proportions of $\mathrm{sp}^{2}$ depending upon the processing conditions[21]. These $\mathrm{sp}^{2}$ bonds create strong intramolecular bonds but also have the potential for attractive intermolecular interactions with polymer chains. Computational and experimental studies have shown that pi-pi stacking can occur between the aromatic structure of styrene and the $\mathrm{sp}^{2}$ bonds of graphene[22, 23]. These bonds are stronger than generic intermolecular forces, and their influences on mechanical properties are the focus of this study.

In this study, interphase properties are investigated on a series of different polymers on a graphitic-like carbon surface. Four polymers were studied: polybutadiene (PBD, $\mathrm{T}_{g}=-50^{\circ} \mathrm{C}$ ), polystyrene $\left(\mathrm{PS}, \mathrm{T}_{\mathrm{g}}=120^{\circ} \mathrm{C}\right.$ ) and two different random solution-styrene butadiene rubbers (SSBR): SSBR1 $\left(T_{g}=5^{\circ} \mathrm{C}\right)$ and SSBR2 $\left(T_{g}=20^{\circ} \mathrm{C}\right)$. These polymers encompass a fairly broad spectrum, spanning rubbery to glassy at room temperature. SSBR is a random copolymer comprised of varying amounts of styrene and butadiene monomers. In this data set, SSBR2 has more styrene content than SSBR1 and a corresponding higher $T_{g}$. PS has phenyl rings on each repeat unit that have the potential to undergo 
significant pi-pi bonding interactions with a graphite-like carbon substrate. The concentration of the phenyl rings is reduced by using styrene-butadiene copolymers and completely eliminated by using PB. Three of these four polymers are rubbery at room temperature (PBD, SSBR1, SSBR2) and one is glassy (PS). These four polymers were chosen to examine the effect of polymer and filler surface chemistry on interphase stiffening effects in the case of styrene containing polymers and graphitic surfaces.

\section{Experimental Methods}

\section{Materials}

Rubbers (SSBR1, SSBR2 and PBD) used in this study were prepared by Goodyear Tire and Rubber Company and used as received. Polystyrene polymer $(\mathrm{Mn}=280,000 \mathrm{~g} / \mathrm{mol})$ was purchased from Pressure Chemical Co. and used as received. Glass transition temperatures reported for each polymer was measured via dynamic mechanical analysis (DMA) and taken as the peak value of tan delta (E'/E') at $1 \mathrm{~Hz}$. Use of ellipsometry to determine $\mathrm{Tg}$ in PS films supported on diamond-like carbon has revealed no difference within error from bulk Tg in films as thin as $50 \mathrm{~nm}$, which is below the thickness of the thinnest film in the current study. Thus, we expect no significant influence of Tg-confinement effects on modulus-confinement effects for the conditions considered in the current study.

\section{Surface Preparation}

Silicon substrates (5 $\mathrm{mm} \times 5 \mathrm{~mm}, 1 \mathrm{~mm}$ thickness) were prepared by cleaning the top surfaces using acetone and lightly rubbing with Kimwipes. Any remaining solvent was evaporated prior to the use of the substrates. Cleaned silica surfaces 
were coated with amorphous carbon using a Deskill Carbon Coater and graphitic rods. Exposure times were approximately $10-20 \mathrm{sec}$ to obtain $4-7 \mathrm{~nm}$ coatings. Spin Coating Polymer Layers

$150 \mathrm{mg}$ of polymer was dissolved in $5 \mathrm{~mL}$ of toluene, and the solution was spun using a vortex stirrer. After the polymer was fully dissolved, several drops of the polymer solution were placed on the top surface of the substrate to nearly cover the entire area. Substrates were spun at approximately 2500 RPM for $45 \mathrm{sec}$ to obtain a flat and uniform film. To obtain thinner films, the polymer solution was diluted using additional toluene.

Any trace levels of solvent was evaporated by holding the films above their glass transition temperature $\left(23^{\circ} \mathrm{C}\right.$ for rubbers and $130{ }^{\circ} \mathrm{C}$ for polystyrene) under vacuum for over $12 \mathrm{hr}$.

\section{Film Characterization- Ellipsometry}

Spectroscopic ellipsometry (M-2000D, J.A. Woollam Co., Inc) was used to measure the deposited carbon layer and polymer film thickness. Because each material has unique optical parameters, a layer model can be used to describe samples stacks and are composed of a number of discrete, homogeneous, welldefined layers. The optical model for the graphitic surface consists of four layers that include the silicon substrate, native oxide layer, the carbon film, and a roughness layer [24-26]. Optical constants supplied by J.A. Woollam Co. were used to represent the silicon and native oxide layers. Ellipsometry measurements on a clean silica wafer confirmed that the native oxide layer present on the surface of the wafers is $2 \mathrm{~nm}$. A B-spline model was used to model the dielectric properties of the carbon layer following a procedure used by Weber et al. [26]. Here, the number of coefficients used in the recursive 
polynomial model needed to accurately describe the dielectric function was maintained as low as possible while still adhering to the shape of the function. The roughness layer was modeled by a Bruggeman's effective medium approximation (EMA) of $50 \%$ bulk material and $50 \%$ voids $[25,26]$. Due to the complexity of the model, profilometry and atomic force microscopy (AFM) were used to confirm the validity of the thickness and roughness and were found to be in good agreement with spectroscopic data. The actual roughness is small; AFM measurements done on sputtered carbon 14-nm-thick yielded a Ra of $0.11 \mathrm{~nm}$. To model the polymer film present on the carbon surface, we introduced a fifth Cauchy transparent model layer.

\section{Surface characterization}

Characterization of the chemical bonding of the surface was performed using $\mathrm{x}$ ray photoelectron spectroscopy (Thermo Scientific ESCALAB $250 \mathrm{Xi)}$. A survey scan (pass energy: $150 \mathrm{eV}$, energy step size: $1 \mathrm{eV}$ over binding energy (B.E) range of 0 to $1200 \mathrm{eV}$ ) was performed using a monochromatic Al Ka x-ray source together with a low-voltage electron flood gun for charge compensation. Fine spectra of $\mathrm{C} 1 \mathrm{~s}$ peaks was collected at $50 \mathrm{eV}$ pass energy and with a resolution of $0.5 \mathrm{eV}$ averaged over 8 scans. XPSPEAK 4.1, a free curve fitting software, was used to fit the C1s curve.

\section{Nanoindentation}

Nanoindentation experiments were performed using a Hysitron Triboindenter and a $100 \mu \mathrm{m}$ radius diamond tip. Before data collection, the machine was allowed to settle by holding a small contact load $(0.1-0.5 \mu \mathrm{N})$ on the top of the film over a period of $10+\min$. This period of time allowed the thermal drift of the machine to 
reach steady state before data collection, which reduces drift during the frequency sweep. After this period of time, the tip was disengaged and moved to a new site for data collection. Loads were set to obtain a quasi-static displacement between $30-60 \%$ of the film thickness and oscillation displacements between 0.5-3.0 nm. Using these parameters, frequency sweeps were obtained between $10-250 \mathrm{~Hz}$.

Temperature modulation was performed using an integrated thermoelectric cooler with a modest argon gas flow to prevent moisture or ice formation on the top of the sample. The sample was allowed $20+$ min to reach steady state.

\section{Creation of FE Models}

Axisymmetric finite element models were created using ABAQUS finite elements package. These models had two different parts: the large spherical tip and the film, a meshed deformable part with quadratic tetragonal elements (C3D4); meshes were refined near contact in the polymer domain to help assist model convergence. Contact between parts were modeled as frictionless and 'hard contact' with a master (diamond tip) and slave surface (polymer). The tip and a substrate were created and meshed as deformable objects. In the models where the modulus was appropriately high, the film is sufficiently stiff that the deformation of the substrate and tip must be included to have an accurate model. Sliding boundary conditions were modeled between the polymer film and supporting silica. This boundary condition was defined because of the magnitude of nanoindentation results and the large Poisson ratio of the films. 
The polymer was assigned elastic viscoelastic properties determined from macroscopic dynamic mechanical analysis (DMA) data on the same material, while the silicon substrate was assigned purely elastic material properties (70 GPa).

\section{Results}

\section{Surface Characterization}

In order to characterize the chemistry of the sputter-coated carbon on the substrate surface, XPS was performed. This method can determine the relative composition of molecules by detecting binding energies associated with specific types of bonds. In our case, we are interested in the presence of hybridized carbon-carbon bonds, $\mathrm{sp}^{2}$. In the case of pure graphene, the surface is comprised of nearly all $\mathrm{sp}^{2}$ bonds. However, because we are using graphitic rods for sputter-coating, we expect to observe measurable amounts of single carbon-carbon bonds, $\mathrm{sp}^{3}$. The $\mathrm{C} 1 \mathrm{~s}$ fine spectrum is shown in Figure 2. The full width at half maximum (FWHM) of the C1s peak in the graphite film is about 1.9 $\mathrm{eV}$ and is much larger than that reported for graphite $(\mathrm{FWHM}=1.0-1.3 \mathrm{eV})$ or for diamond $(F W H M=1.1-1.4 \mathrm{eV})[27,28]$. Because of this, the substrate is assumed to have both $\mathrm{sp}^{2}$ and $\mathrm{sp}^{3}$ configurations, and the $\mathrm{C} 1 \mathrm{~s}$ peak can be fitted with two major peaks. A curve fitting tool was used to separate the contributions of the $\mathrm{sp}^{2}$ and $\mathrm{sp}^{3}$ bonding configurations as well as any chemisorbed (CO) species. The peak location of the first component is located at $284.0 \mathrm{eV}$ (FWHM $=1.16 \mathrm{eV}$ ) and corresponds to the $\mathrm{sp}^{2}$ atoms, while the second peak is at 284.7 $\mathrm{eV}(\mathrm{FWHM}=1.2 \mathrm{eV})$ and corresponds to the $\mathrm{sp}^{3}$ peak. A third peak of smaller intensity is seen at $\sim 285.7 \mathrm{eV}$ and corresponds to $\mathrm{C}-\mathrm{O}$ contamination due to exposure to air. According to the peak split areas, the carbon layer contains $52 \%$ $\mathrm{sp}^{3}$ and $48 \% \mathrm{sp}^{2}$. The presence of the $\mathrm{sp}^{2}$ bonds is important to the resulting 
interphase properties, potentially creating sites for pi-pi stacking with phenyl rings in styrene monomers.

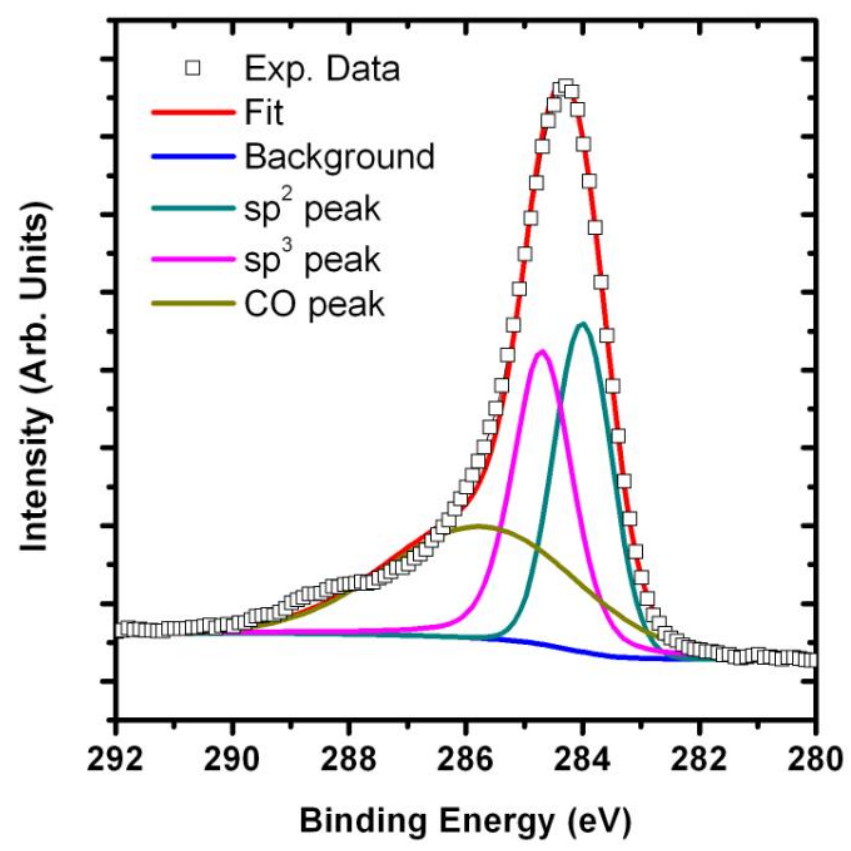

Figure 2. XPS results shows that the carbon surface contains a $0.52: 0.48$ ratio of $\mathrm{sp}^{3}$ to $\mathrm{sp}^{2}$ bonds, as well as some $\mathrm{C}-\mathrm{O}$ bonds. The hybridized $\mathrm{sp}^{2}$ bonds present opportunities for pi-pi stacking with the aromatic structure in styrene repeat units. Although these bonds are slightly outnumbered by single carbon-carbon bonds, there are present in sufficient quantity to provide opportunities for these types of interactions.

\section{Removal of Substrate Effects}

The influence of the hard substrate is critical in thin polymer film nanoindentation experiments, especially with large radius tips. Even at low displacements for moderate thickness films, the hard substrate that supports the film greatly impacts the results. Figure 3 demonstrates this substrate effect with a $100 \mu \mathrm{m}$ radius tip on films of varying thicknesses determined by finite element (FE) models using the Oliver/Pharr model for oscillatory loading [29] (see Supporting Information). In this study, large contact areas were needed to increase the load 
necessary for shallow indents on rubber thin films. Due to the size and shape of the spherical tip, substrate effects manifest differently than Berkovich or cubecorner tips. As shown in Figure 3, simple finite element models without any special interactions show that even at very shallow indentations $(>5 \%)$, the measured modulus is much higher than the input modulus; ideally, when the sample is infinitely thick, indentation returns the input modulus with a ratio of 1.0 . We see that as the film thickness increases, we approach this ideal condition. 

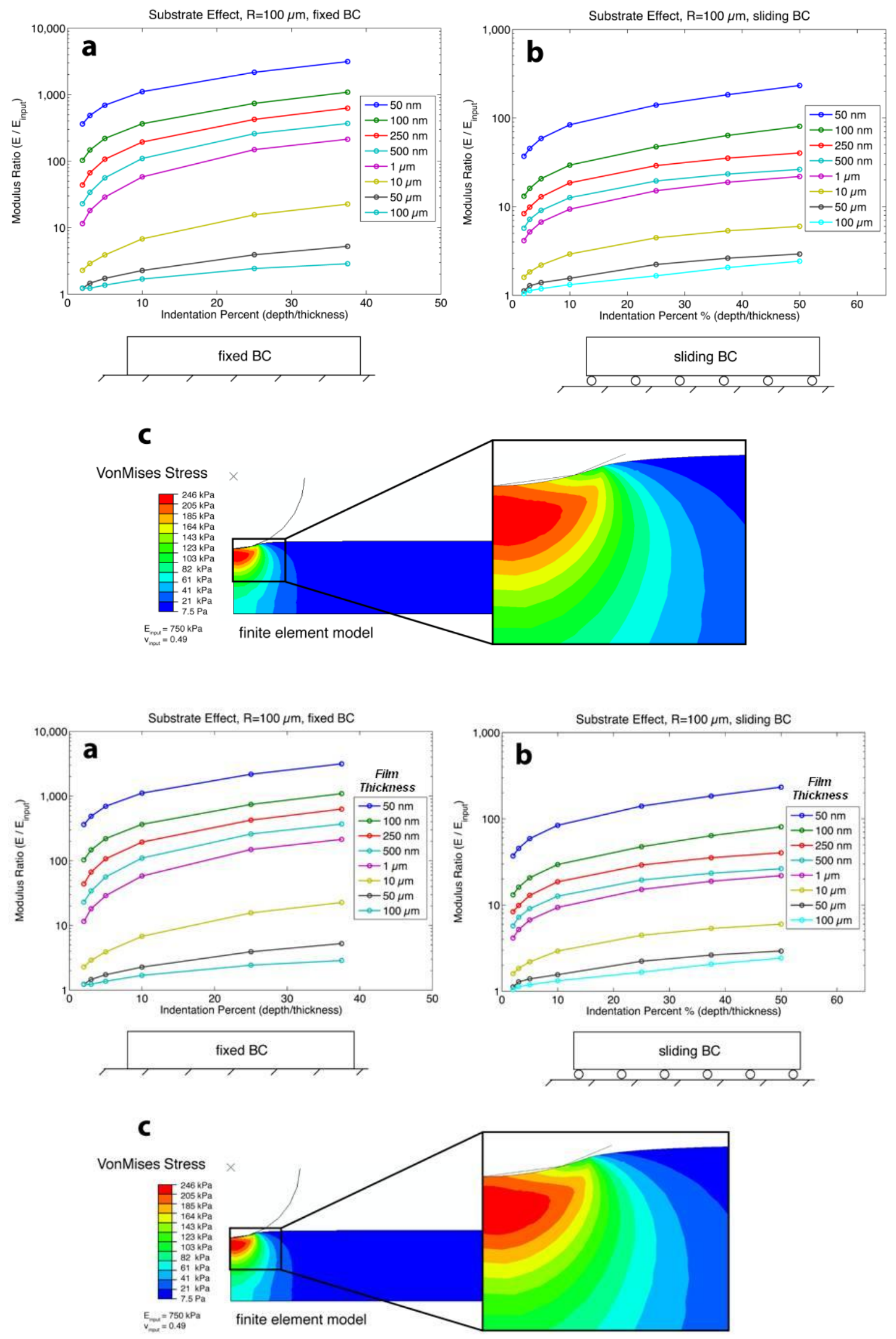
Figure 2. Substrate effect trends for a large rigid spherical tip, $100 \mu \mathrm{m}$ radius, on thin films of varying thicknesses (1 MPa) are displayed for either fixed (a) or sliding (b) conditions of the bottom of the film. The results show that the substrate effect shape is concave and grows immediately at low contact depths and more slowly as the depth is increased into the film. FE visualization displays the corresponding stress gradients existing below the tip with a large spherical tip (c) and the interaction of those stress fields with the substrate.

This substrate effect has varying magnitude depending upon the contact depth of the film and has a concave shape. The differences between Figures 3a and $3 \mathrm{~b}$ demonstrate that the lower boundary condition of the polymer layer impacts the resulting substrate effect. In the case of a fixed film, Figure 3a, we see that if the film is firmly attached and cannot freely expand to the substrate effect is intensified due to the Poisson effect. A similar trend develops when the film is only physically supported and allowed to slide along the bottom, Figure $3 \mathrm{~b}$. The substrate effect must be removed from experimental results to draw proper comparisons of the influence of the interphase for varying film thicknesses and indentation depths. Therefore, equivalent FE models were created to match experimental conditions such as film thickness, frequency, contact depth, oscillatory displacement, bulk material properties and film attachment.

\section{Nanoindentation Results}

In order to compare results from various samples, which have different magnitudes of modulus, a method must be developed to equally compare the results. Additionally, due to the presence of the hard substrate, measured modulus values can depend upon the indentation depth. Therefore, we normalize the experimental results with FE model results to remove the effects of the substrate. These normalizations assume that the FE models properly match all 
relevant conditions (substrate influences, bulk polymer modulus), solely with the exception of interphase contributions. Equation 1 defines the normalization for the storage modulus, and Equation 2 does the same for tan(delta):

$$
\begin{aligned}
& R\left(h_{c}, h_{o s c}, f, t\right)=E_{\text {exp }} / E_{F E} \\
& R\left(h_{c}, h_{o s c}, f, t\right)=\tan ()_{\exp } / \tan ()_{F E}
\end{aligned}
$$

In these equations, the ratios are defined as functions of contact depth $\left(h_{c}\right)$, oscillation displacement $\left(h_{o s c}\right)$, frequency $(f)$ and film thickness $(t)$ - as well as any physical conditions created by model assembly. If experimental results match the FE models, these terms become unity (1.0) and represent experimental cases with little interphase effect. In the case when positive interphase gradients are present, these terms will grow in magnitude. The experimental results are standardized from films of varying thicknesses in order to make proper comparisons between samples and indentation ratios. As observed in Figure 3, the substrate effect is highly sensitive to both the film thickness and indentation depth into the film. With the experimental method, the measured modulus is an effective modulus which depends upon both the bulk and interphase polymer probed by the nanoindenter. As the film thickness decreases, the effective or measured modulus is more influenced by the interphase emanating from the substrate. More detailed results for direct measurement of interphase modulus, and the gradient of modulus away from the surface, can be extracted through a modified setup [30].

For each of these polymers, three films were tested: a thick film on silica, a thick film on carbon and a thin film of the same chemistry on carbon. Silica was 
chosen as our base substrate due to its inorganic composition and reflectivity, important for ellipsometry. Additionally, one of the SSBR1 samples was cooled to $0{ }^{\circ} \mathrm{C}$ to investigate how the interphase changes near the glass transition temperature. If interphase effects are present, there should be an increase in normalized modulus from thick to thin films, as the interphase becomes a larger portion of the whole film.

Collected results from the polymer film nanoindentation contain several important findings. In order to display those findings efficiently, we summarize them in Figure 4 (full data sets are provided in the Supporting Information). Optical micrographs of all samples tested are provided, displayed in Figure 4a, to display the surface quality of each sample. Storage modulus (stiffness) and tan

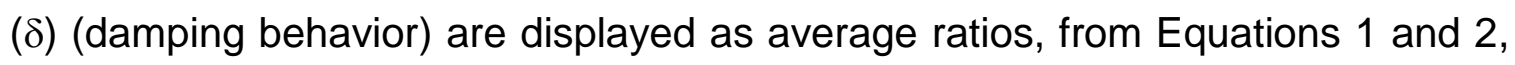
across the testing frequency range $(10-250 \mathrm{~Hz})$ and as bar graphs in Figure 4b-c. 

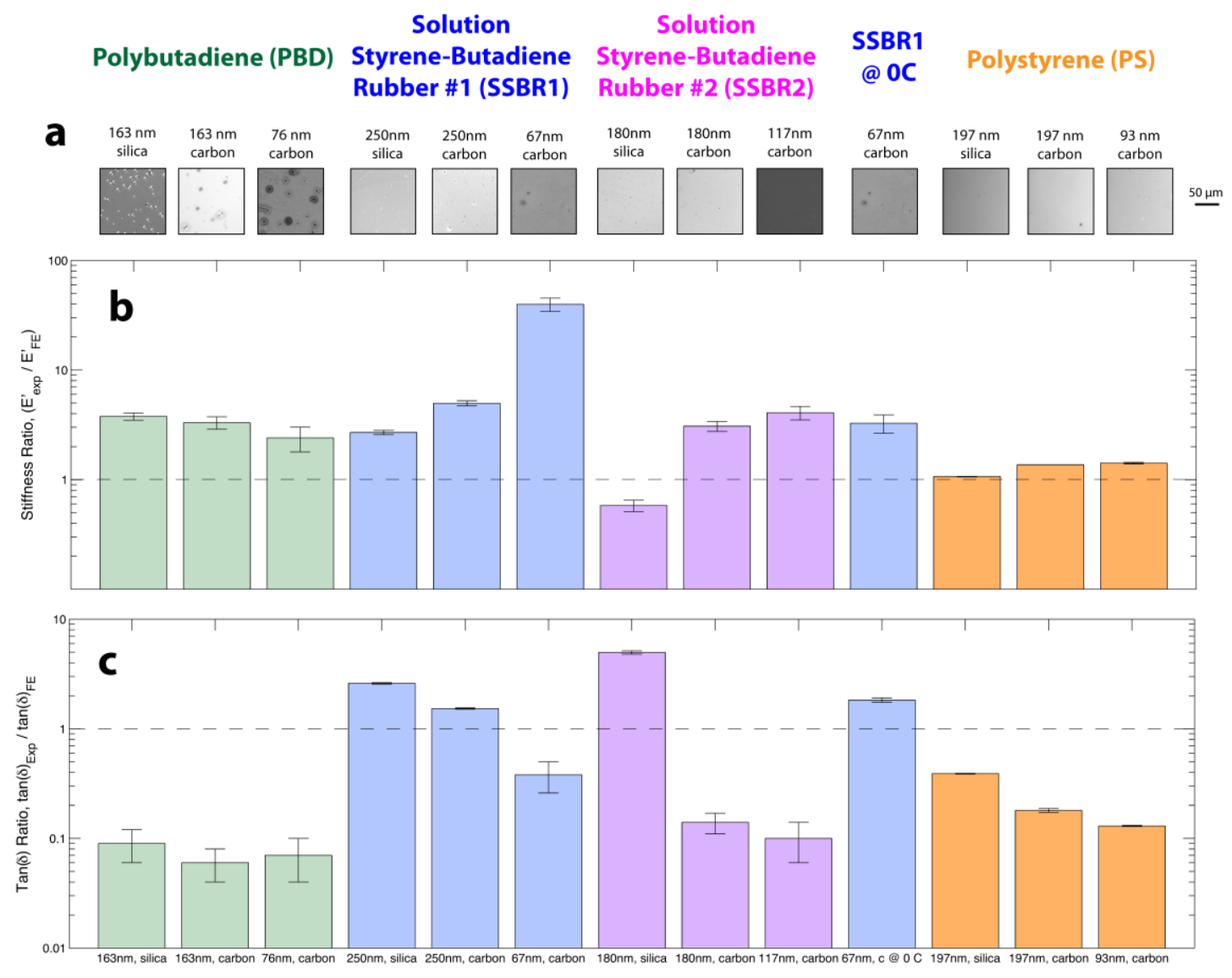

Figure 4. Optical micrographs (a) of thin films (top-down view through polymer to reflective substrate) are displayed as well as summarized stiffening results ( $f=100$ $\mathrm{Hz}$ ), which shows changes in film stiffness/modulus (E') for polymer films on silica and carbon surfaces (b). In optical micrographs, dewetting is noticeable in the polybutadiene films. Deviations of the results from unity (1), indicate stronger contributions from the interphase for those samples. ,Samples in the rubbery domain show more stiffening effects than samples near or in the glassy state. Three of the four polymers demonstrate stiffening effects as the sample thickness is reduced, indicating stronger influence from the proportionally larger interphase in those samples. The $\tan (\delta)$ response is also plotted (c) showing how the damping properties commensurately reduce in tandem with stiffening of $E$ '.

\section{Discussion}

The summarized results in Figure 4 display a comprehensive view of polymer and substrate interactions. In the case of PBD (green), there is little dependence on either thickness or surface chemistry for both stiffness and damping effects, as each bar graph with associated standard deviations overlap. In Figure 4a the optical micrographs for PBD displayed noticeable voiding and 
dewetting, which indicates a lack of significant polymer-substrate attractive interactions [31]. The results indicate that there is a stiffening effect (E' 2-3 fold increase) on all of the three surfaces (4b) and a corresponding drop in the damping response (4c). This set of results from PBD is interpreted as simple physical confinement of the polymer, induced by the silica or carbon substrate, coupled with the free surface effect, which has the overall effect of increasing the modulus.

With the two SSBR polymers (blue and purple) we see stronger interphase effects. Unlike the data from PBD, the optical micrographs show well wetted films in which we observe clean optical micrographs with good adhesion and little or no voiding between substrate and polymer, and corresponding changes in the stiffness from sample to sample. For both types of SSBR, we observe increases in stiffness when we (a) introduce a carbon substrate and (b) reduce the thickness of the polymer film. The first increase (from the first to second bar in each sequence) is associated with attractive polymer-substrate chemical interactions between the polymer and the substrate as carbon replaces silica. As discussed previously, aromatic structures in styrene repeat units are able to pi-pi stack with graphitic surfaces, specifically $\mathrm{sp}^{2}$ bonds. These pi-pi interactions are stronger than generic van der Waals forces and therefore could influence interphase enhancements. These enhancements similarly grow when the thickness of the sample in reduced (second to third bar in sequence), providing greater sensitivity to the stronger portions of the interphase gradient. As the stiffness increases in these cases (E' 3-50 fold increase), it comes at the 
cost of the damping properties (Figure 4c). The results provide evidence that the polymer in the interphase region is confined with reduced mobility and enhanced rigidity and thus increased stiffness and reduced damping.

The results from these three different rubbers demonstrate that polymer composition and the polymer-substrate interfacial interactions influence the interphase properties. However, the dependency of polymer state, either glassy or rubbery, on the interphase gradients is likewise an important consideration in obtaining a more encompassing view of interphase behavior. We have initial comparisons of this effect, by comparing SSBR1 to SSBR2; SSBR2 has a higher $T_{g}$, near the testing temperature. The interphase enhancements are reduced for the film close to the glass transition regime, SSBR2, compared to the film in the rubbery state, SSBR1.

In order to provide alternate ways to study interphase behaviors as the rubber becomes glassy, additional samples were tested. First, a thin SSBR1 sample on carbon was reduced to a lower temperature $\left(0^{\circ} \mathrm{C}\right)$ to simulate a semiglassy response - i.e., in the transition region between glassy and rubbery response. The reduction in temperature decreases the stiffening effect ( $E$ ' reduces from a 50 to a 3 fold increase) in Figure $4 \mathrm{~b}$ and compares nicely to the results seen for SSBR2 on a carbon surface. These results indicate that interphase enhancement depend upon the state, or bulk mobility, of the polymer.

Due to the limitations on our experimental set-up, the true glassy responses of our rubbers were not accessible. Therefore, we further studied this polymer state influence by investigating PS (orange). PS contains the same 
styrene repeat unit to which we attribute the positive interphase effects as in the SSBR rubbers but is in the glassy state at room temperature. Three PS samples were tested through the method outlined for PBD and SSBR samples. Normalized results from PS show positive interphase effects on a carbon surface (1.3-1.4 fold increase in E'), that are of a significantly lower magnitude. The low interphase enhancements, along with SSBR1 and SSBR2 results, indicate that interphase stiffness enhancements reduce as the bulk polymer mobility reduces and are consistent with AFM data on glassy polymers [30].

A potential limitation of contact based methods is the tip-surface effects that might influence results. Researchers have shown that the tip adhesion as well as free-surface effects or created interphase from the tip contact $[32,33]$ can influence results. In this study, we demonstrate interphase effects from graphitic surfaces against a control surface, silica. Through the design of the experiment, the chemistry of the polymer at the tip is identical for each polymer type and indentation depths are comparable; therefore, complicated effects at the tippolymer surface are reduced, allowing for comparisons between samples of different thickness. For the types of loads in this study $(\sim 10 \mathrm{uN})$ combined with a theoretical strong attraction $\left(0.05 \mathrm{~J} / \mathrm{m}^{2}\right)$, the purely elastic Johnson-KendallRoberts model (JKR) [34] predicts the measured modulus would be about 5 times the actual modulus. Experimental demonstrations of the JKR adhesion effects show that beyond $10 \mathrm{~nm}$ indentation depth (for a given tip geometry) these influences are, in fact, negligible [35]. Future extraction of tip effects could potentially change the overall magnitude of these results and allow for more 
precise comparisons between polymer types; however, we conclude that the higher level qualitative trends would be preserved.

\section{Conclusions}

Styrene-based polymers have shown significant modulus enhancements (up to 50 fold) for thin films on graphitic surfaces through nanoindentation. We demonstrate that as chemical interactions strengthen, these enhancements grow. The modulus enhancements with styrene-based polymers can be linked to the potential pi-pi stacking between the aromatic units in styrene repeat units and the $\mathrm{sp}^{2}$ bonds in amorphous carbon. These bonds are stronger than van der Waals forces and create a strong attraction between the polymer chains and interface. In the case of PBD, we attribute the modest modulus increases to purely physical confinement on the polymer at the interface with no chemical contributions. Overall, the confinement makes the polymer more rigid, simultaneously increasing the stiffness (E') and reducing the damping properties $(\tan (\delta))$

Interphase enhancements also depend upon the state of the polymer. As the rubber transitions to the glassy state, the bulk mobility of the chains reduces which inhibits the interphase enhancements. The results imply that some lower limit exists from which mobility cannot be further reduced. In the case of glassy polymers, this minimum (a maximum modulus) is closer to the bulk state of the polymer than when it is rubbery. This interphase enhancement dependency on bulk polymer mobility could be explained by many different phenomena, such as a rigid amorphous fraction [36] at the interface; however, more study is required 
to make conclusions of that nature. The data contained within this study demonstrates several important findings about interphase behavior: interphase enhancements depend upon both (i) physical confinement and (ii) attractive interfacial interactions, as well as (iii) bulk polymer mobility.

Future work with focus on varying testing temperatures can provide a more complete picture about how bulk polymer mobility affects interphase gradients. Additionally, more sophisticated computational models will be developed to extract interphase characteristics, such as length and magnitude, from indentation results.

\section{Acknowledgements}

Support for this project was provided by Goodyear Tire and Rubber Company (Akron, $\mathrm{OH}$ ) and by the National Science Foundation under grant number CMMI1235355. The nanoindentation measurements were carried out in the NIFTI facility of NUANCE Center at Northwestern University. The NUANCE Center is supported by NSF-NSEC, NSF-MRSEC, Keck Foundation, the State of Illinois, and Northwestern University. Optical images were generated in the Optical Microscopy and Metallography Facility at Northwestern.

\section{References}

[1]. De Greef, N., et al., The effect of carbon nanotubes on the damage development in carbon fiber/epoxy composites. Carbon. 49(14): p. 4650-4664.

[2]. Huang, Y., et al., Particle Matrix Interfacial Bonding - Effect on the Fracture Properties of Rubber-Modified Epoxy Polymers. Advances in Chemistry Series, 1993(233): p. 189-210.

[3]. Ramanathan, T., H. Liu, and L.C. Brinson, Functionalized SWNT/polymer nanocomposites for dramatic property improvement. Journal of Polymer Science Part B-Polymer Physics, 2005. 43(17): p. 2269-2279. 
[4]. Ramanathan, T., et al., Graphitic nanofillers in PMMA nanocomposites-An investigation of particle size and dispersion and their influence on nanocomposite properties. Journal of Polymer Science Part B: Polymer Physics, 2007. 45(15): p. 2097-2112.

[5]. RamanathanT, et al., Functionalized graphene sheets for polymer nanocomposites. Nat Nano, 2008. 3(6): p. 327-331.

[6]. Hamming, L.M., et al., Effects of dispersion and interfacial modification on the macroscale properties of TiO2 polymer-matrix nanocomposites. Composites Science and Technology, 2009. 69(11-12): p. 1880-1886.

[7]. Qiao, R. and L. Catherine Brinson, Simulation of interphase percolation and gradients in polymer nanocomposites. Composites Science and Technology, 2009. 69(3-4): p. 491-499.

[8]. Ellison, C.J. and J.M. Torkelson, The distribution of glass-transition temperatures in nanoscopically confined glass formers. Nature Materials, 2003. 2(10): p. 695-700.

[9]. Rittigstein, P., et al., Model polymer nanocomposites provide an understanding of confinement effects in real nanocomposites. Nature Materials, 2007. 6(4): p. 278-282.

[10]. Yang, Z.H., et al., Glass Transition Dynamics and Surface Layer Mobility in Unentangled Polystyrene Films. Science, 2010. 328(5986): p. 1676-1679.

[11]. Fakhraai, Z. and J.A. Forrest, Measuring the surface dynamics of glassy polymers. Science, 2008. 319(5863): p. 600-604.

[12]. Bodiguel, H. and C. Fretigny, Viscoelastic properties of ultrathin polystyrene films. Macromolecules, 2007. 40(20): p. 7291-7298.

[13]. Miyake, K., N. Satomi, and S. Sasaki, Elastic modulus of polystyrene film from near surface to bulk measured by nanoindentation using atomic force microscopy. Applied Physics Letters, 2006. 89(3).

[14]. Stafford, C.M., et al., Elastic moduli of ultrathin amorphous polymer films. Macromolecules, 2006. 39(15): p. 5095-5099.

[15]. Wood, C.D., et al., Nanoscale structure and local mechanical properties of fiberreinforced composites containing MWCNT-grafted hybrid glass fibers. Composites Science and Technology, 2012.

[16]. Lv, P., et al., Increasing the interfacial strength in carbon fiber/epoxy composites by controlling the orientation and length of carbon nanotubes grown on the fibers. Carbon, 2011.49(14): p. 4665-4673.

[17]. Qu, M., et al., Nanoscale visualization and multiscale mechanical implications of bound rubber interphases in rubber-carbon black nanocomposites. Soft Matter, 2011. 7(3): p. 1066-1077.

[18]. Watcharotone, S., et al., Interfacial and Substrate Effects on Local Elastic Properties of Polymers Using Coupled Experiments and Modeling of Nanoindentation. Advanced Engineering Materials, 2011. 13(5): p. 400-404.

[19]. Zhou, B. and B.C. Prorok, A new paradigm in thin film indentation. Journal of Materials Research, 2010. 25(9): p. 1671-1678.

[20]. Zhou, B. and B.C. Prorok, A Discontinuous Elastic Interface Transfer Model of Thin Film Nanoindentation. Experimental Mechanics, 2010. 50(6): p. 793-801.

[21]. Robertson, J., Diamond-like amorphous carbon. Materials Science \& Engineering RReports, 2002. 37(4-6): p. 129-281.

[22]. Su, Y.H., et al., Electrostatic studies of pi-pi interaction for benzene stacking on a graphene layer. Applied Physics Letters, 2011.99(16).

[23]. Shen, B., et al., Melt Blending In situ Enhances the Interaction between Polystyrene and Graphene through pi-pi Stacking. Acs Applied Materials \& Interfaces, 2011. 3(8): p. 3103-3109. 
[24]. Dey, R.M., et al., Diamond like carbon coatings deposited by microwave plasma CVD: XPS and ellipsometric studies. Bulletin of Materials Science, 2007. 30(6): p. 541-546.

[25]. Weber, J.W., V.E. Calado, and M.C.M. Van de Sanden, Optical constants of graphene measured by spectroscopic ellipsometry. Applied Physics Letters, 2010. 97(9): p. 091904-091904-3.

[26]. Weber, J.W., et al., B-spline parametrization of the dielectric function applied to spectroscopic ellipsometry on amorphous carbon. Journal of Applied Physics, 2009. 106(12): p. 123503.

[27]. Leung, T. Y., et al., Determination of the sp 3 / sp 2 ratio of a-C : H by XPS and XAES J. Non. Cryst. Solids 1999. 254(1), p. 156-160..

[28]. Merel, P., et al., Direct evaluation of the sp 3 content in diamond-like-carbon films by XPS. Applied Surface Science, 1998. 136(1): p. 105-110.

[29]. Loubet, J., B. Lucas, and W. Oliver, Some measurements of viscoelastic properties with the help of nanoindentation, in International Workshop on Instrumental Indentation, D.T. Smith, Editor 1995: San Diego, CA. p. 31-34.

[30]. Cheng, X., et al., Characterization of Local Elastic Modulus in Confined Polymer Films via AFM Indentation. Macromolecular rapid communications, 2014.

[31]. Barnes, K.A., et al., Suppression of dewetting in nanoparticle-filled polymer films. Macromolecules, 2000. 33(11): p. 4177-4185.

[32]. Carrillo, F., et al., Nanoindentation of polydimethylsiloxane elastomers: Effect of crosslinking, work of adhesion, and fluid environment on elastic modulus (vol 20, pg 2820, 2005). Journal of Materials Research, 2006. 21(2): p. 535-537.

[33]. Kohn, J.C. and D.M. Ebenstein, Eliminating adhesion errors in nanoindentation of compliant polymers and hydrogels. Journal of the Mechanical Behavior of Biomedical Materials, 2013. 20: p. 316-326.

[34]. Johnson, K.L., K. Kendall, and A.D. Roberts. Surface energy and the contact of elastic solids. in Proceedings of the Royal Society of London A: Mathematical, Physical and Engineering Sciences. 1971. The Royal Society.

[35]. Cheng, X., Characterization of local mechanical properties of polymer thin films and polymer nanocomposites via AFM indentations., 2014, Northwestern University: Available from Dissertations \& Theses @ CIC Institutions; Dissertations \& Theses @ Northwestern University.

[36]. Xu, H. and P. Cebe, Heat capacity study of isotactic polystyrene: Dual reversible crystal melting and relaxation of rigid amorphous fraction. Macromolecules, 2004. 37(8): p. 2797-2806. 


\section{Supporting Information}

\section{Material Models}

An important component of this study was the creation of finite element models. In these models, equivalent bulk material properties determined via dynamic mechanical analysis were applied to the finite element models. These material models, displayed in Figure 1, were determined using TTSP over a range of -100 $\mathrm{C}$ to $50 \mathrm{C}$ at low strain $(0.05 \%)$. For polystyrene, a glassy polymer, the properties at room temperature were applied to the model, shown in Figure 2.
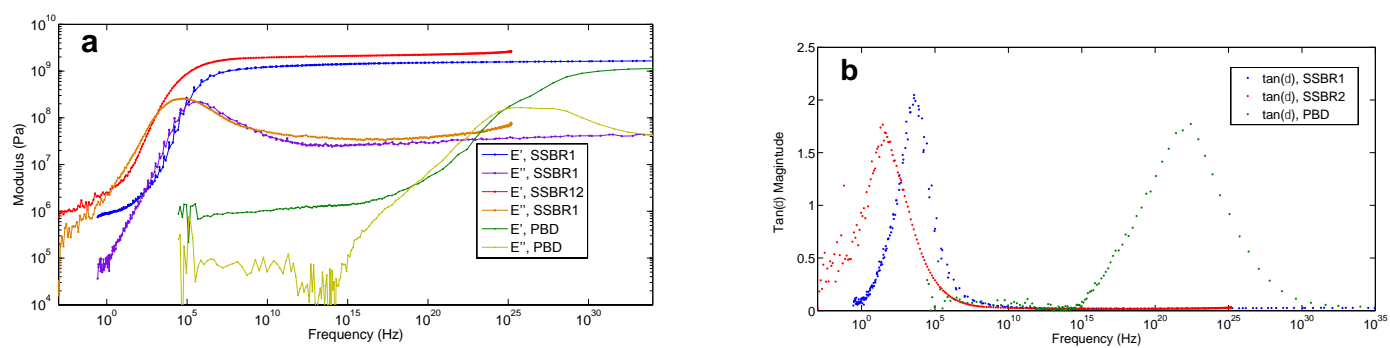

Figure 1. Frequency dependent mechanical properties used for defining the material model for rubbers in finite element models. From these two images, it is shown the range of different glass transition response the three rubbers have. From this image, we can see that the two SSBR samples have similar tan(delta) responses, however the SSBR2 has a higher $\mathrm{Tg}$ (inversely proportional to frequency).

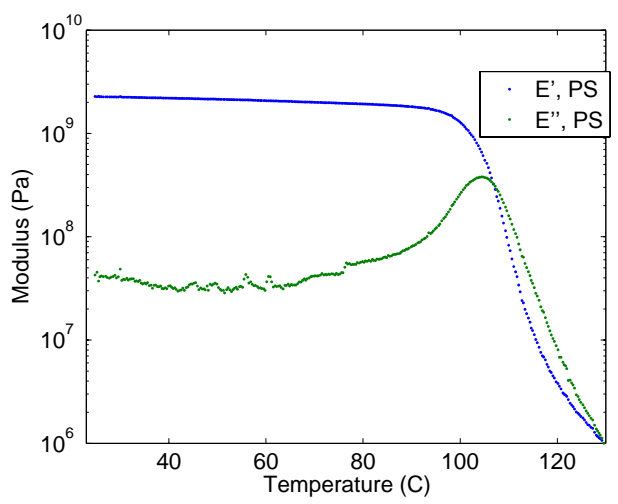

Figure 2. Temperature sweep data from polystyrene demonstrate glassy response at room temperature. Therefore, frequency modulation in nanoindentation $(10-250 \mathrm{~Hz})$ does not greatly change the mechical response of the polymer. 
In order to appropriately match experimental data, finite element models were created with three different parts: the indentation tip, the polymer film and the supporting silica substrate. These models proved to be more accurate than those with rigid supports for this application.

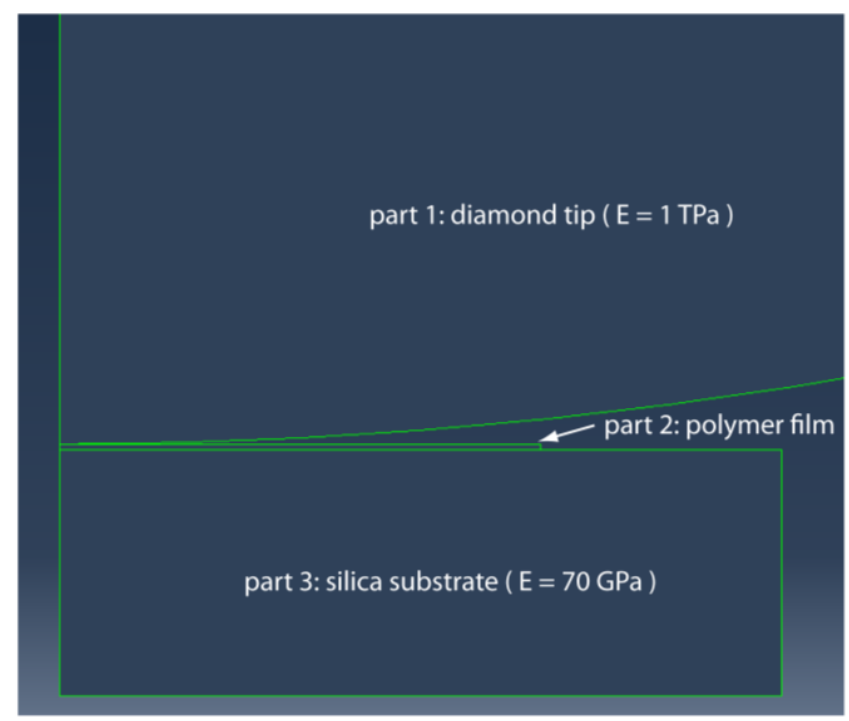

Figure 3. In finite element models of experiments, three separate parts were modeled: the tip, the polymer film and the silica substrate. The film was allowed to slide freely on top of the silica (frictionless) during indentation. This schematic modeled our experimental conditions better than with a rigid support, which create very large increases in apparent stiffness of the polymer film.

\section{Dynamic Indentation Mechanics}

The formulation for dynamics indentation properties starts with a differential equation that defines the motion of a oscillating mass with a given force amplitude. These fairly commonly used relationships were initially defined by Loubet et al. [29] and is integrated into the Hysitron analysis software. This equation will define the motion of the indenter when in contact with the material of interest with some stiffness, $\mathrm{k}$, and damping, C, response. In this case, the 
steady-state response of the displacement due to the oscillating force, which share a common frequency.

$F_{0} \sin (t)=m \ddot{x}+C \dot{x}+k x$

$x=X \sin (t+)$

Next, these equations are rearranged to solve for stiffness, k, and damping, C, coefficients as a function of force, displacement, phase offset and frequency.

$$
\begin{aligned}
& k=\frac{F}{X} \frac{1}{\sqrt{1+\tan ^{2}}}+m^{2} \\
& C=\sqrt{\frac{(F / X)^{2} \tan ^{2}}{1+\tan ^{2}}} \frac{1}{}
\end{aligned}
$$

These coefficients define the stiffness and damping of the total motion, which includes the tip and material of interest. Therefore, to isolate the material response from the total response, the stiffness and damping are broken down into contributions of the material and the tip.

$$
\begin{aligned}
& k_{s}=k \quad k_{i} \\
& C_{s}=C \quad C_{i}
\end{aligned}
$$

Finally, the complex modulus is defined as a function of the stiffness and damping relationships.

$E^{*}=(E+i E)=\frac{S^{*} \sqrt{ }}{2 \sqrt{A_{c}}}=\frac{\left(\begin{array}{ll}k+i C & C\end{array}\right)}{2 \sqrt{A_{c}}}$

\section{Nanoindentation Frequency Sweeps}


Through our indentation process, we were able to collect information about the E' and the E" portions of the complex modulus through frequency sweeps from 10$250 \mathrm{~Hz}$. The averaged and summarized results are displayed in the body of the text in Figure 4. Below, we display the raw frequency sweeps for each sample, however averaged across three independent tests at different sites at the film.
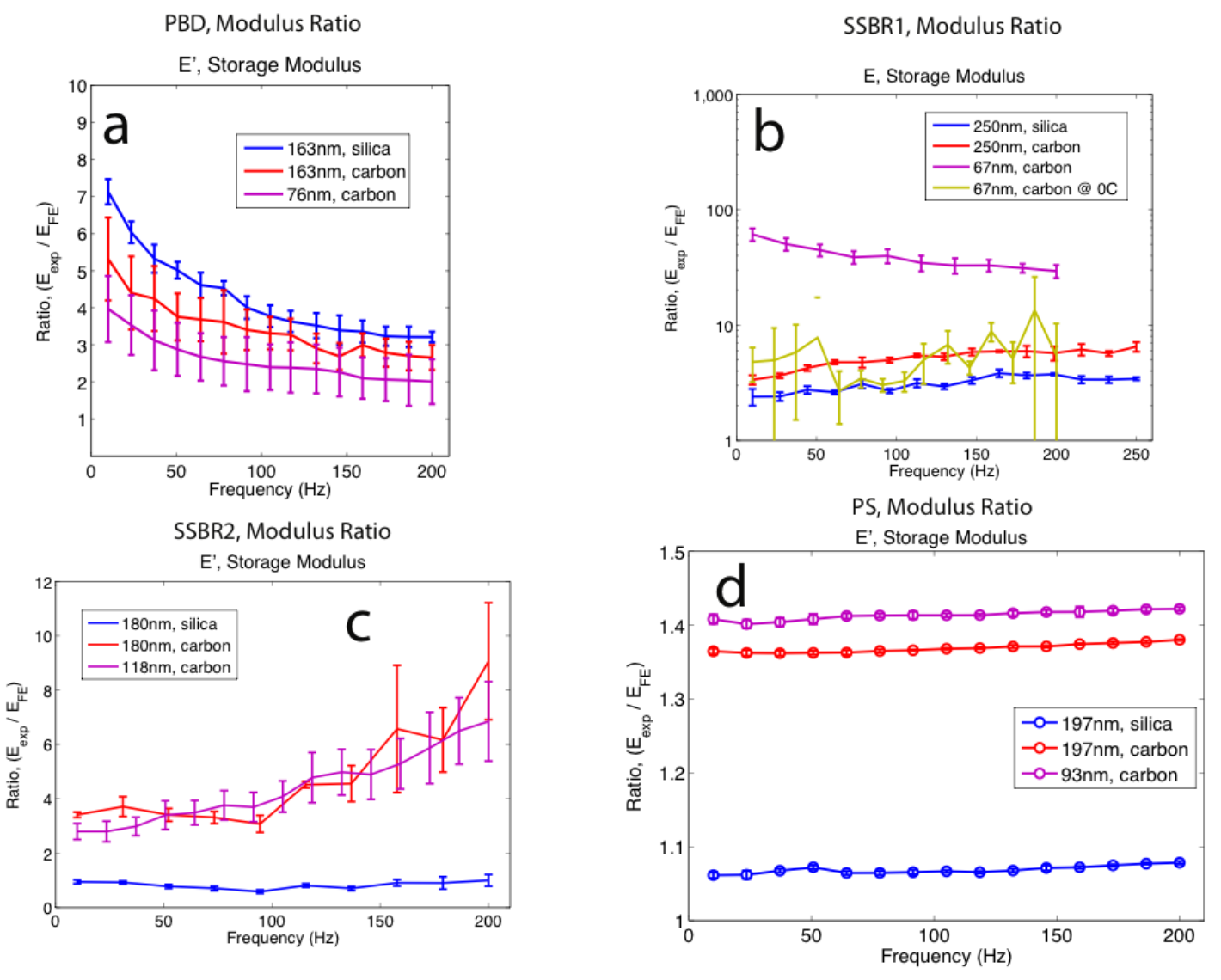

Figure 4. Storage Modulus ratios plotted as a function of probing frequency for PBD (a), SSBR1 (b), SSBR2 (c) and PS (d). 

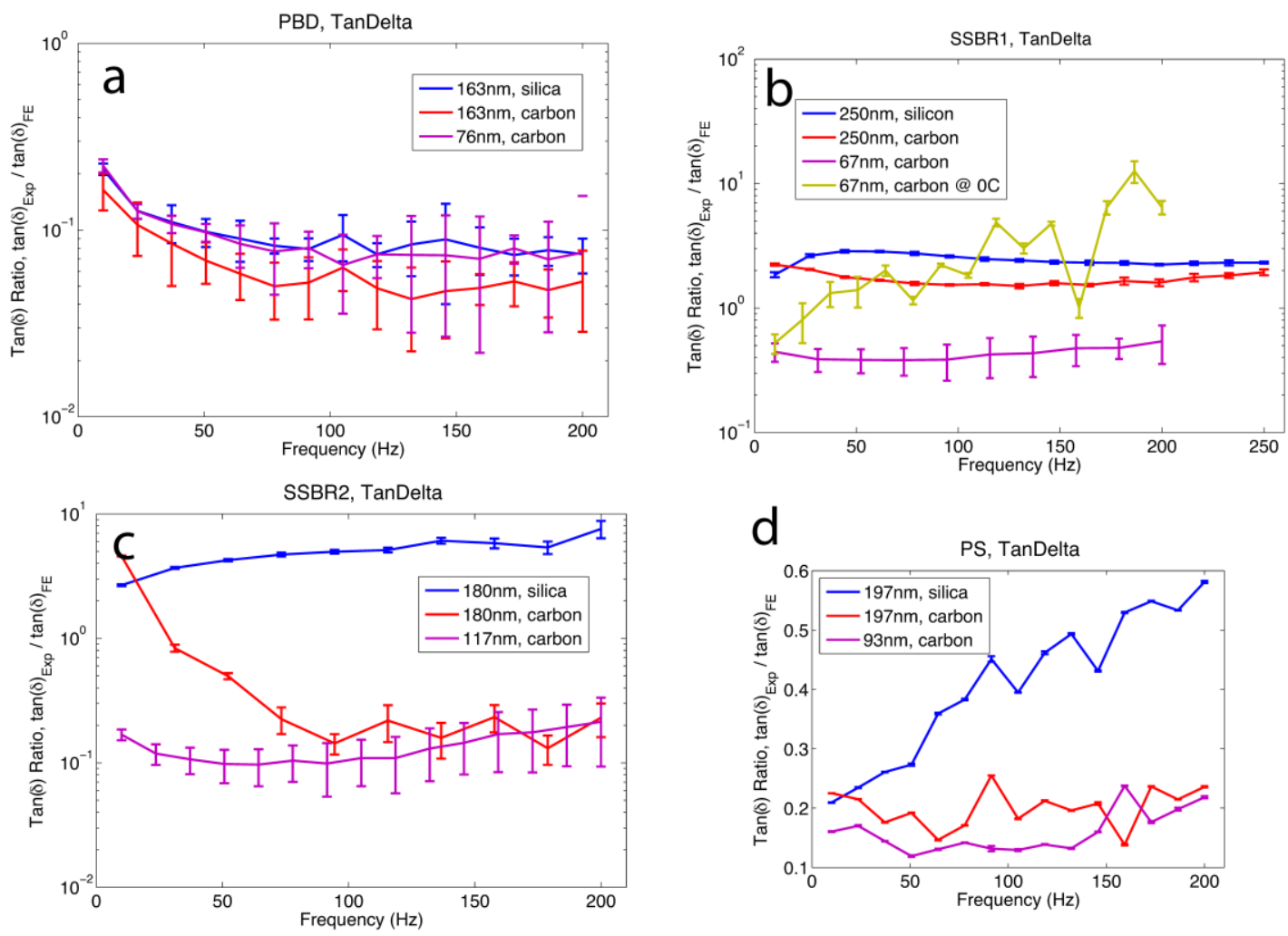

Figure 5. TanDelta ratios plotted as a function of probing frequency for PBD (a), SSBR1 (b), SSBR2 (c) and PS (d). 

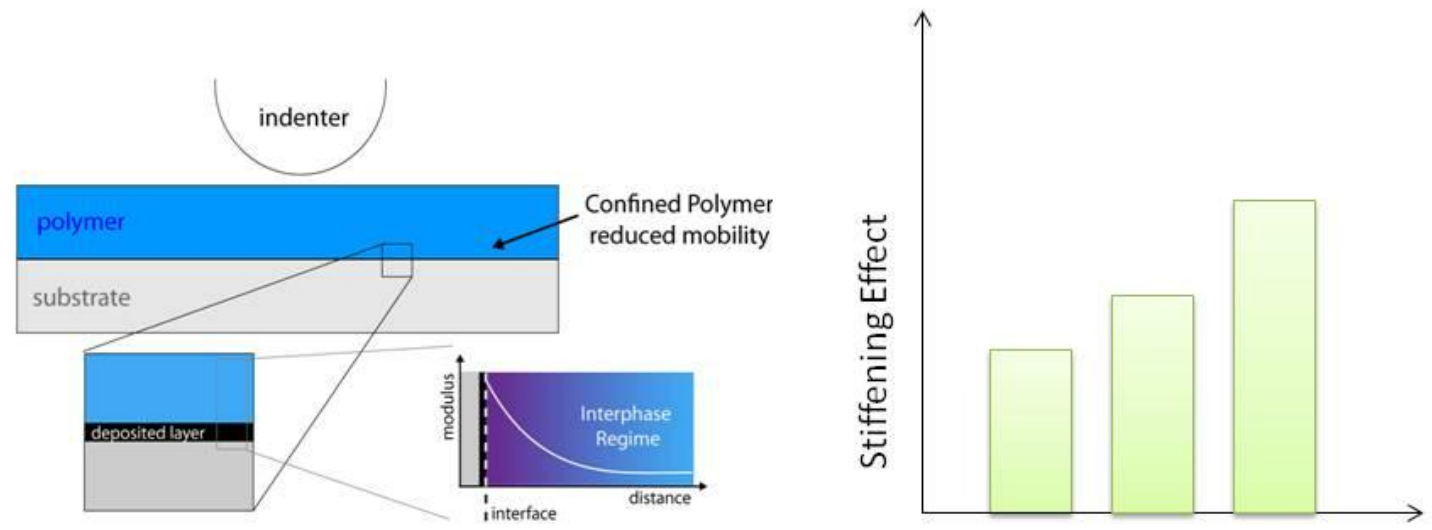

Film thickness $\downarrow$ 